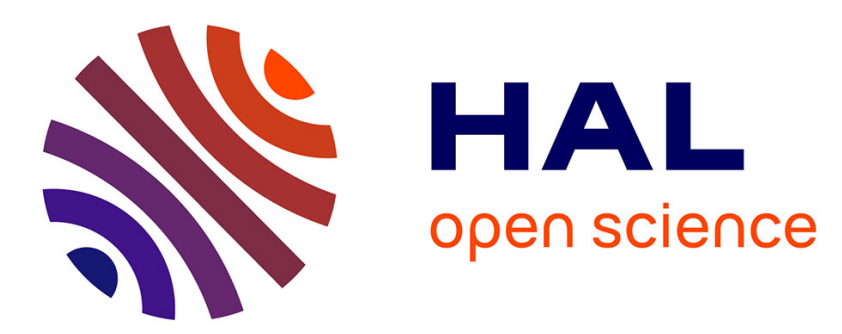

\title{
Analyse de quelques concepts en cartographie des sols basée sur une automatisation des cartes thématiques dérivées
}

\author{
Dominique King
}

\section{- To cite this version:}

Dominique King. Analyse de quelques concepts en cartographie des sols basée sur une automatisation des cartes thématiques dérivées. Agronomie, 1984, 4 (5), pp.461-472. hal-00884660

\section{HAL Id: hal-00884660 https://hal.science/hal-00884660}

Submitted on 1 Jan 1984

HAL is a multi-disciplinary open access archive for the deposit and dissemination of scientific research documents, whether they are published or not. The documents may come from teaching and research institutions in France or abroad, or from public or private research centers.
L'archive ouverte pluridisciplinaire HAL, est destinée au dépôt et à la diffusion de documents scientifiques de niveau recherche, publiés ou non, émanant des établissements d'enseignement et de recherche français ou étrangers, des laboratoires publics ou privés. 


\section{Analyse de quelques concepts en cartographie des sols basée sur une automatisation des cartes thématiques dérivées}

Dominique KING

I.N.R.A., Service d'Etude des Sols et de la Carte Pédologique de France, Centre de Recherches d'Orléans, Ardon, F 45160 Olivet

L'expression cartographique des données de sols pose le problème de la perte de l'information liée aux limites des techniques graphiques. L'auteur propose une mémorisation informatique globale de la carte pédologique et de sa notice, chaque unité cartographique étant décrite sur la base d'un éventail de caractères le plus large possible. Les essais de thématisation de 2 cartes pédologiques mettent en évidence l'importance d'une description de la variabilité à l'intérieur même de chacune des unités cartographiques élémentaires. Avec chaque carte thématique, est proposée une carte de la variabilité intra-unité donnant une première appréciation du niveau de précision auquel le pédologue, auteur de la carte, s'est situé. Cette méthode devrait permettre la valorisation des documents pédologiques tout en évitant des interprétations trop schématiques des documents thématiques.

Mots clés additionnels : Pédologie, informatique, thématique, précision cartographique, banque de données. maps.

The problem of the cartographic expression of soil data is the loss of information arising from limits in graphic technique. The author proposes a fully computerized storage system for the pedological map and its notice, each cartographic unit being described on the basis of as many characteristics as possible. Attempts to " thematize » two pedological maps in this way showed the importance of describing the variability within each elementary cartographic unit. Each thematic map was accompanied by a map of intra-unit variability, giving a first impression of the level of accuracy at which the pedological mapper was working. This method should allow one to computerize pedological data without making a too sketchy interpretation of thematic documents.

Additional key words : Pedology, computerized data processing, thematic, cartography precision, data bank, variability.

\section{INTRODUCTION}

Le sol se présente au niveau géographique comme un milieu continu et variable. Le pédologue cartographe tente de cerner cette variabilité et de comprendre les relations existant entre celle-ci et les autres éléments du milieu. La synthèse de son travail s'exprime sous la forme d'une carte accompagnée d'une notice. Nous pouvons donc définir la carte pédologique comme un modèle synthétique et explicatif de la répartition des sols d'une région (JAMAGNE et al., 1977).

Un tel document contient de nombreuses informations concernant la morphologie et la genèse des sols ainsi qu'un diagnostic sur leur fonctionnement et des pronostics sur leur comportement. L'auteur du document pédologique cherche à représenter sur la carte le plus grand nombre possible de caractères à l'aide de divers moyens graphiques. Trop de surcharges par des couleurs et des sigles rendent alors difficile la lecture du document. D'ailleurs, en dépit de ces efforts, pour des raisons d'échelle, de contraintes graphiques ou de coûts, certaines données se trouvent consignées seulement dans la notice. Aussi l'utilisateur ne peut pas avoir rapidement une vue synthétique de la répartition spatiale d'un seul ou de plusieurs paramètres combinés entre eux. Ces combinaisons peuvent être simples, par exemple sols calcaires et profonds, ou très complexes, par exemple modélisation de la réserve en eau des sols, potentialités agricoles, simulations de scénarios d'amé- 
nagement... Dans tous les cas, cela nécessite un travail long, fastidieux voire impossible.

Avec le développement des techniques informatiques sont apparus divers systèmes se proposant de stocker sous forme numérique les données contenues dans les cartes pédologiques (LYNCH, 1976 ; BIE \& SCHELLING, 1977 ; Garbouchev \& SAdovski, 1978 ; Mc CorMACK et al., 1978) et de valoriser ainsi le document pédologique à des fins d'utilisations diverses (FALQUE, 1975 ; JOHNSON, 1975 ; DOMPTAIL, 1977 ; FORD \& NIELSEN, 1978 ; HAANS \& VAN LYNDEN, 1978 ; BOON, 1981). De nombreuses méthodes proposent une mémorisation de chacun des points d'observations ayant servi à l'établissement de la carte, constituant ainsi des " banques de données-sol " (par exemple, en France : VAN DEN DRIESSCHE, 1974 ; BERTRAND et al., 1979 ; ACCT, 1979) auxquelles peuvent être adjoints des programmes d'interpolation de façon à construire automatiquement des cartes (DOUGENIK \& SHEENAN, 1975 ; MALlet, 1976 ; AUBRY et al., 1980). Mais, peu de systèmes proposent une informatisation complète et synthétique de la carte pédologique, du fait des contraintes techniques liées à la saisie des contours pédologiques (VAN ZUIJLEN, 1975 ; TOMLINSON, 1978 ; BURROUGH, 1980 ; VAN KUILENBURG et al., 1981 ; VOSS, 1981). Nous avons décrit précédemment les diverses méthodes utilisées pour la saisie de documents graphiques (KING, 1981). Une des solutions proposées par certains auteurs (par exemple, LEGROS \& HENSEL, 1977 ; RAGG, 1977 ; TRUSZKOWSKA et al., 1981) consiste à établir un maillage systématique de la carte et à caractériser chaque morceau de la couverture pédologique ainsi virtuellement découpée. En procédant manuellement, on est contraint à limiter le temps de saisie ; il faut alors choisir une maille assez grossière aux dépens de la précision des limites cartographiques. Nous avons donc cherché à automatiser cette saisie afin de réduire la maille d'enregistrement à des dimensions très petites compatibles avec la précision souhaitée.

Du fait de ces contraintes techniques, peu d'auteurs se sont intéressés au problème de la description informatisée d'unités cartographiques de sols (CORMACK, 1976 ; JANSEN \& ARNOLD, 1976). Pourtant, la carte pédologique ne doit pas être assimilée au seul document graphique ; en conséquence, nous proposons une méthode permettant la mémorisation informatique du maximum d'informations contenues dans la notice (voire également des informations non transcrites et connues seulement de l'auteur de la carte). Il est, en effet, indispensable qu'une thématisation en aval de la carte des sols s'appuie, d'une part, sur un ensemble de données le plus complet possible et tienne compte, d'autre part, de la complexité de l'organisation spatiale du sol : superposition des horizons, unités complexes, variations en continu des caractères, variations décelées mais non reconnues géographiquement...

Nous présentons dans une première partie les techniques informatiques mises en place puis, sur la base d'expériences de thématisations de 2 cartes pédologiques, nous discutons les résultats obtenus, en examinant plus particulièrement les conséquences sur la codification de la variabilité du sol, sur l'élaboration de la carte pédologique et sur la validité des documents produits.
II. MÉTHODE UTILISÉE (SYSTÈME KALEIDOS)

\section{A. Analyse informatique de la carte pédologique : Hiérarchisation de l'information}

Une carte pédologique peut se décomposer en une partie graphique, représentant le "contenant » et matérialisée par le dessin des contours, et une autre partie, dite sémantique, représentant le «contenu » et se rattachant plus particulièrement à la légende et à la notice. Le plus petit élément de surface contenant de l'information est la plage cartographique définie par un contour continu, fermé sur lui-même (Nous n'étudierons pas les autres éléments informationnels d'une carte que sont le point et la ligne. L'information « point » se rattache aux banques de données sols classiques. L'information « ligne » peut préciser graphiquement le contraste existant entre deux plages cartographiques juxtaposées (GIRARD, 1976)). Plusieurs plages peuvent avoir les mêmes caractéristiques et former une unité cartographique dont nous proposons la définition suivante :

L'unité cartographique (U.C.) est un (ou des) morceau $(x)$ de la zone étudiée, délimitée graphiquement sur une carte par un (ou des) contour(s) fermé(s). Ces morceaux forment une « unité » dans le sens où l'on ne peut pas - oulet l'on ne veut pas - les subdiviser, à l'échelle de représentation choisie, en sous-ensembles cartographiables.

On ne peut pas subdiviser une U.C. lorsque la contrainte graphique est telle que le dessin devient illisible. On fixe un seuil de représentabilité en suivant par exemple la loi du quart (CRUICKSHAND, 1972 ; BOULAINE, 1980).

On ne veut pas subdiviser une U.C. lorsque la variabilité et la complexité du milieu sont telles qu'on ne veut, eu égard à l'échelle du travail, effectuer un nombre d'observations suffisamment élevé pour délimiter des sous-ensembles (BAIZE, comm. pers., 1982).

L'unité cartographique présentée précédemment forme une unité au sens graphique du terme (même couleur ou même trame). La véritable signification d'une telle unité se trouve dans la notice, au travers d'une autre unité que nous nommerons unité sémantique de sols.

En nous plaçant aux moyennes et grandes échelles, nous pouvons distinguer 2 cas :

Si l'ensemble des sols (des pedons) présente des développements de profils semblables, nous le nommons unité simple de sols (ou unité de sols). Dans ce $1^{\text {er }}$ cas, nous trouvons, sur l'ensemble de cette unité sémantique, la même succession d'horizons principaux (définition de la série de sols, CPCS, 1967). Cependant, nous admettrons des variations sur un nombre limité de caractères.

Dans le $2^{\mathrm{e}}$ cas, l'unité sémantique peut contenir plusieurs unités de sols, nous l'appelons complexe de sols. Celui-ci forme une unité informationnelle d'ordre supérieur à l'unité de sols (fig. 1).

Enfin chaque unité simple de sols se présente sous la forme d'un volume que l'on peut découper en horizons principaux. Cette dernière unité d'information qu'est l'horizon " cartographique » forme un sous-ensemble hiérarchique de l'unité de sols d'ordre inférieur (fig. 1). 


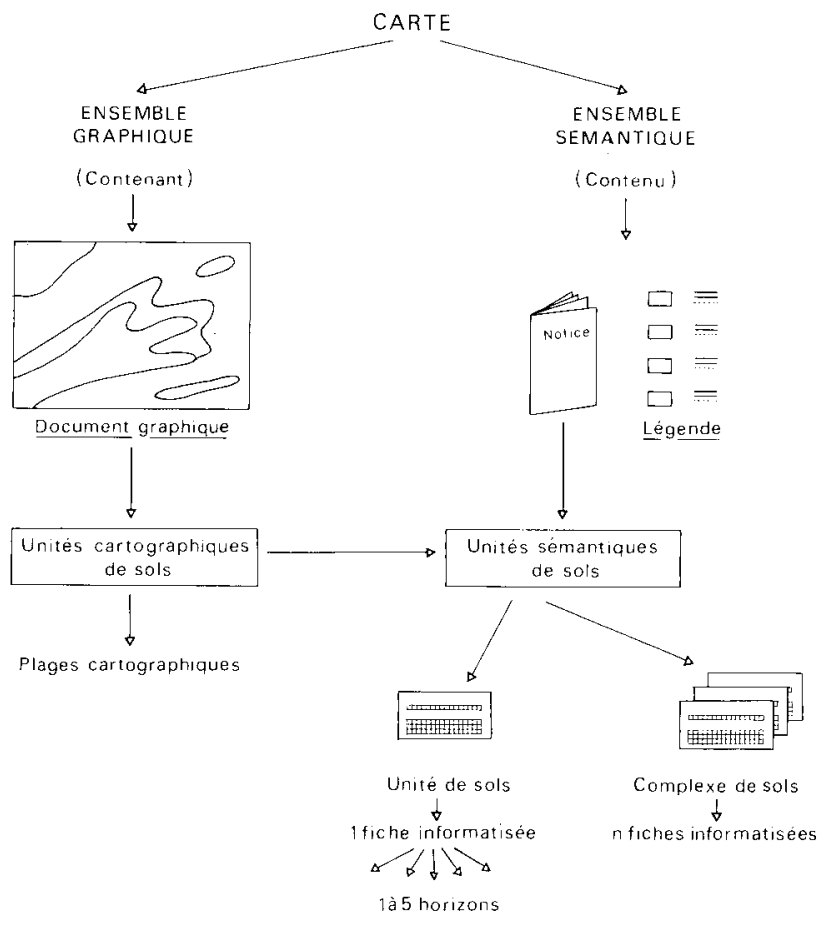

Figure 1

Récapitulation sur les notions de plage et d'unité cartographique (ensemble graphique) et sur les notions d'unité et de complexe de sols (ensemble sémantique).

Recapitulation of the notions of the limit and cartographic unit (graphic system) and the notions of unit and complex of soils (semantic system).

\section{B. Saisie et stockage de l'information}

Nous avons conçu le logiciel KALEIDOS en le segmentant en 2 groupes indépendants de programmes informatiques. Le $1^{\text {er }}$ groupe traite de la partie graphique, le $2^{e}$ de la partie sémantique de la carte.

\section{Logiciel graphique}

Rappelons brièvement les particularités de KALEIDOS dans le domaine graphique (cf. KING, 1984). Le document cartographique est saisi automatiquement à l'aide d'un microdensitomètre optique à balayage. On obtient ainsi une « image numérique » dans laquelle on reconnaît par programme chaque plage cartographique délimitée par un contour fermé. Plusieurs plages cartographiques constituant une même unité de sol sont affectées d'un même identificateur faisant référence à une fiche de description établie par le pédologue (voir ci-dessous). D'autres programmes permettent des mises à jour rapides pour la suppression ou l'ajout d'un contour.

\section{Logiciel « sémantique » : présentation de la fiche de description des unités de sols}

Parallèlement à cette opération, chaque unité de sols est numérotée par le pédologue et décrite à l'aide d'une fiche informatisée (fig. 2).

Notre premier objectif est de concevoir une fiche respectant la hiérarchisation de l'information pédologique. Toutes les fiches décrivant les unités de sols appartenant à un même complexe possèdent un numéro commun (nous indiquons l'estimation $\mathrm{du}$ pourcentage de surface au sein de ce complexe) ; chaque unité de sols peut être décrite en la subdivisant en sous-ensembles correspondant aux horizons principaux.

Notre second objectif est de limiter au mieux la perte d'informations liée à la synthèse faite par le pédologue sur la multitude de ses observations. L'unité de sols est souvent considérée comme " homogène »; elle ne l'est qu'en référence à la précision que l'on s'est fixée pour chacun des caractères descriptifs, en liaison avec l'échelle du document cartographique définitif. En théorie, chaque caractère d'une unité de sols devrait
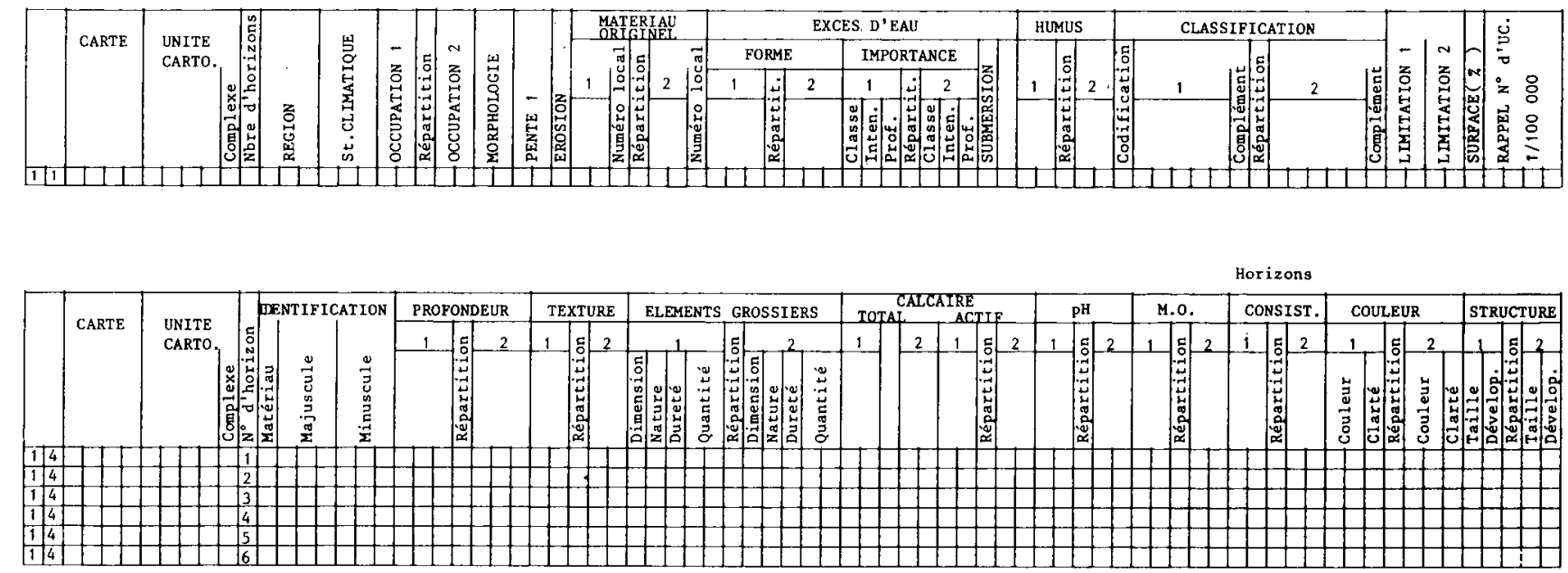

Profils de référence (STIPA)

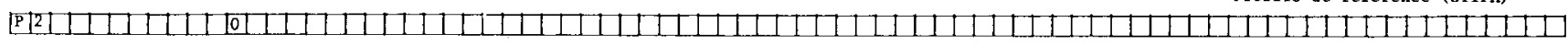

COMMENTAIRES (non pris en compte informatiquement) 
être affecté d'une valeur unique modale, la plus représentative de l'ensemble des observations. En pratique, il est souvent plus facile de donner un intervalle correspondant à la variation observée au sein de chaque unité de sols ; chaque caractère de la fiche a donc été doublé afin de permettre une telle notation. L'ensemble des codes pour chacun des caractères est décrit dans un glossaire afin d'harmoniser la description sur la totalité de la carte (KING, 1982). Nous verrons dans la $3^{e}$ partie comment nous avons été amené à préciser cette notion de "variabilité intra-unité cartographique ".

Le $2^{e}$ groupe de programmes du logiciel KALEIDOS contrôle et gère la partie sémantique c'est-à-dire l'ensemble des fiches de description. Il établit la liaison avec la numérotation automatique de l'ordinateur et calcule, à partir du document graphique enregistré, la surface occupée par chaque unité de sols. Il est possible enfin d'éditer littéralement la description de chaque unité de sols.

\section{Traitement de l'information et expression des résultats}

Nous pouvons remarquer que partie graphique et partie sémantique sont informatiquement indépendantes. En conséquence, nous pouvons traiter séparément ces 2 ensembles.

Nous nous occupons en premier lieu de la partie sémantique en choisissant un caractère et en recherchant les différentes valeurs prises par ce caractère (ainsi que les surfaces respectives correspondantes, en pourcentage ou en hectares, le nombre de plages cartographiques concernées et le rapport de la surface sur le périmètre des contours donnant une idée de la « compacité » des plages). Il est alors possible de faire des regroupements pour des valeurs qui nous semblent proches et d'établir les combinaisons avec d'autres caractères.

Ce type de traitement est simple à mettre en œuvre par le logiciel KALEIDOS et fournit rapidement des résultats sous forme de tableaux statistiques. C'est seulement au vu de ces résultats que nous décidons de la sortie cartographique en s'aidant des programmes de traitements graphiques souvent plus longs et plus coûteux à l'exécution : changement d'échelle, suppression ou épaississement de contours, suppression des unités trop petites (RogalA, 1982), édition définitive sur imprimante graphique. Les normes choisies pour l'enregistrement du document cartographique permettent une restitution sans perte de définition sur la précision des limites pédologiques.

\section{RÉSULTATS DE DIVERS ESSAIS DE THÉMATISATION DE CARTES PÉDOLOGIQUES}

\section{A. Présentation des zones testées}

Deux extraits de cartes pédologiques à l'échelle du 1/50 000 ont été choisis en vue de tester la méthode. Le premier occupe la région du Noyonnais au nord-est du département de l'Oise (BEGON et al., 1977). Cette cartographie s'inscrit dans une étude plus large de ce département. Nous en avons sélectionné un secteur pour la variété des sols rencontrés; en effet, une morphologie accidentée recoupe sur près de $150 \mathrm{~m}$ de dénivelé, divers matériaux géologiques eux-mêmes recouverts d'un dépôt limoneux peu épais. Cette variété des sols explique en grande partie une agriculture également très diversifiée.

Le $2^{e}$ essai s'inscrit dans le cadre du projet des " cartes départementales des terres agricoles 》 (HARDY et al., 1982). Seules les zones agricoles font l'objet d'une cartographie pédologique, bois et friches étant exclus. La variabilité très rapide des caractères des sols fait de cette carte un objet d'étude intéressant pour l'expression cartographique de thèmes à vocation agronomique.

\section{B. Exemples de traitements et d'éditions de cartes thématiques}

Etant donné la souplesse de notre système quant à la codification des caractères descriptifs des unités de sols, il nous est nécessaire, avant tout traitement, d'établir la liste exhaustive de tous les codes employés pour ces caractères.

Par exemple, dans le cas de la carte de la «texture générale de surface du canton de Noyon », nous utilisons un programme informatique de sélection qui dresse la liste des 91 codes utilisés. Ce chiffre important résulte du fait que nous avons donné parfois 2 codes pour une même unité présentant une hétérogénéité interne et que nous avons tenu compte des unités complexes (également double codification, voire triple et plus pour une même unité cartographique).

Par ce programme, nous pouvons établir un regroupement de l'information indispensable à toute synthèse. Il nous donne le nombre total d'unités cartographiques avec le nombre d'unités de sols correspondantes, suivi de l'édition d'un bilan statistique du pourcentage de surfaces d'occupations de chaque unité cartographique. Enfin, pour des codages ayant un sens approché, nous affectons des trames identiques aboutissant ainsi à 15 unités de texture représentées sur la figure 3 .

\section{Les sorties graphiques}

A titre d'illustration, nous nous sommes limité à 2 exemples intéressant la valorisation des cartes pédologiques. Le $1^{\text {er }}$ exprime la texture des horizons de surface (fig. 4). Il s'agit d'une thématique simple ne faisant intervenir qu'un seul paramètre. Le $2^{\mathrm{e}}$ exemple porte sur la réserve en eau des sols (fig. 6) ; il est évidemment plus complexe puisque le calcul implique la combinaison de plusieurs caractères : texture de chaque horizon principal, profondeurs des horizons, charge en éléments grossiers, nature du matériau sousjacent...

Dans le cas où l'unité de sols est caractérisée par une seule valeur modale, aucun problème ne se pose pour l'expression cartographique de cette valeur. Par contre, si l'unité est caractérisée par un intervalle de variation, il nous a semblé important de signaler l'ampleur des variations ou leur nature par des sorties cartographiques (fig. 5 et 7 ), de façon à attirer l'attention de l'utilisateur des documents thématiques sur la variabilité possible intra-unité ou sur notre incertitude dans 


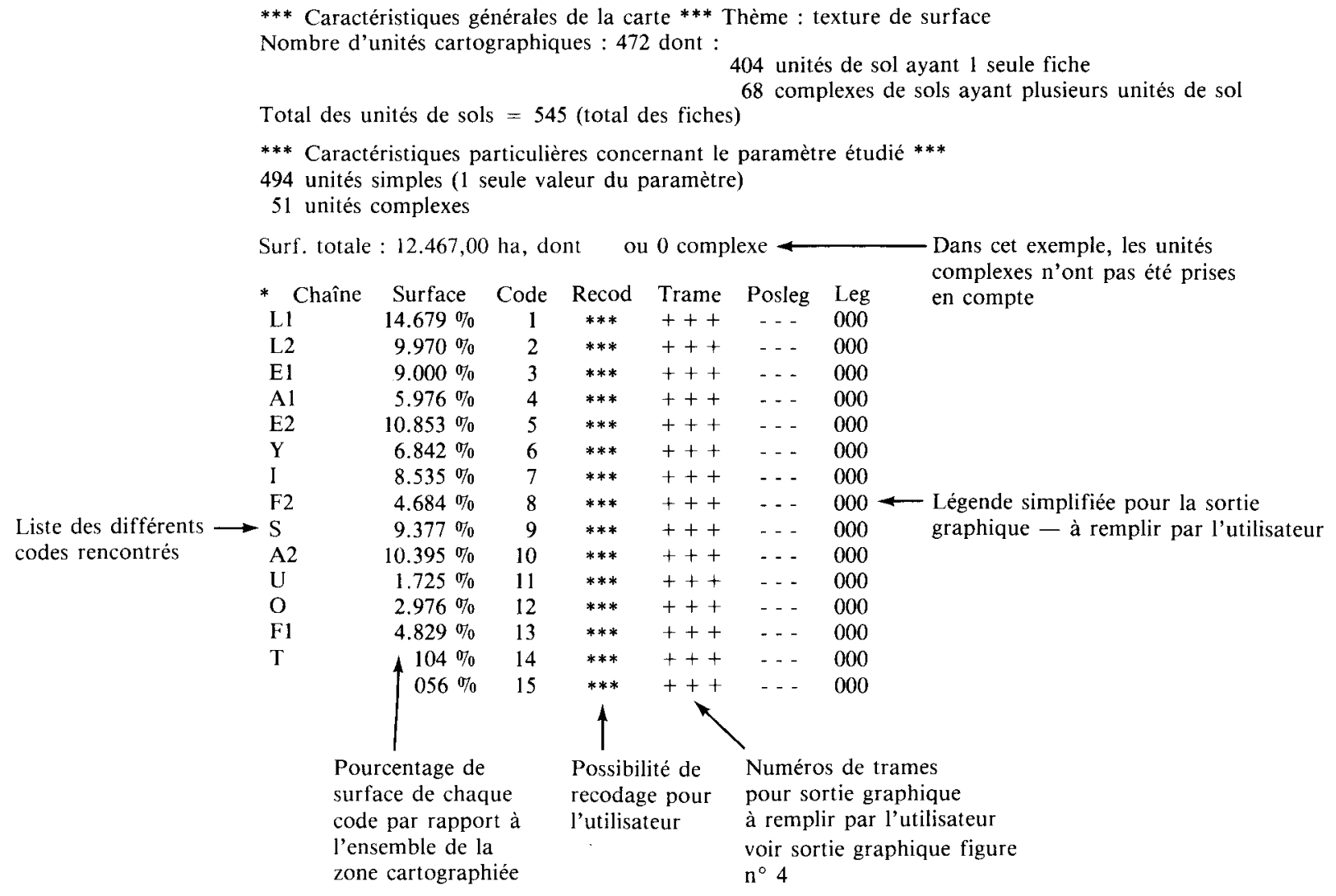

Figure 3

Exemple de sélection " thématique » à partir des fiches de description des unités cartographiques de sols.

certaines zones complexes. Nous développons dans la $3^{\text {e }}$ partie les possibilités d'expression de cette variabilité intra-unité.

\section{DISCUSSIONS ET CONSÉQUENCES SUR L'ÉLABORATION DE LA CARTE DES SOLS}

\section{A. Importance de l'informatisation au cours de l'éla- boration cartographique}

Lorsque le pédologue dessine les contours définitifs sur sa minute de terrain, il synthétise l'ensemble de ses observations et est en mesure de définir précisément les unités cartographiques qu'il met en évidence. $L$ 'informatisation de la carte pédologique doit donc se faire au cours de cette élaboration.

Le choix des variables de caractérisation des unités de sols est essentiel et doit être réfléchi d'une façon approfondie par l'auteur de la carte. Les caractères de description des unités sont, tout d'abord, les critères d'observations morphologiques ayant servi à la cartographie : texture, charge en cailloux de surface, couleur... etc. ; ces données peuvent ensuite être complétées par des données de type analytique.

Il s'agit de ne pas réduire la description d'une unité spatiale de plusieurs ha, voire de plusieurs $\mathrm{km}^{2}$, à la description d'un profil de référence. Le sol se présente sous la forme d'un continuum ; il faut donc le caractériser en tant que tel et faire appel à l'ensemble de nos connaissances de terrain et de laboratoire ainsi qu'aux
Thematic selection example of from index-cards of description of cartographic units of soils.

documents de base (photographies aériennes, cartes topographiques... etc.) pour caractériser ces unités de sols sans méconnaître leur hétérogénéité.

L'édition automatique de cartes thématiques nous permet de visualiser correctement les phénomènes étudiés. On peut être amené ainsi à modifier l'estimation de certains caractères. En ce sens, l'informatisation de la carte pédologique participe à l'élaboration définitive du document cartographique.

\section{B. Synthèse " thématique " des données et intérêt de la méthode pour la mise en place de modèles}

Plus nous prévoyons de possibilités d'expression des caractères, plus il devient difficile d'éditer un document graphique synthétique simple à utiliser. Dans le cas de thématiques faisant intervenir plusieurs caractères, on est obligé de suivre une démarche identique à celle présentée précédemment si on ne veut pas être submergé par la multitude des combinaisons possibles. Nous avons donc complété notre logiciel sémantique initial par des possibilités simples de recodage et par la mémorisation systématique de ces regroupements. Ceux-ci peuvent être différents pour un même caractère, suivant les thèmes et les particularités que l'on désire faire ressortir. Cette façon de procéder peut sembler lourde mais il est préférable de "perdre" l'information en toute connaissance de cause, au moment de notre réflexion sur un thème donné, plutôt que de ne jamais la voir apparaître à cause d'une synthèse cartographique commencée trop tôt au cours de la construction de la carte. 


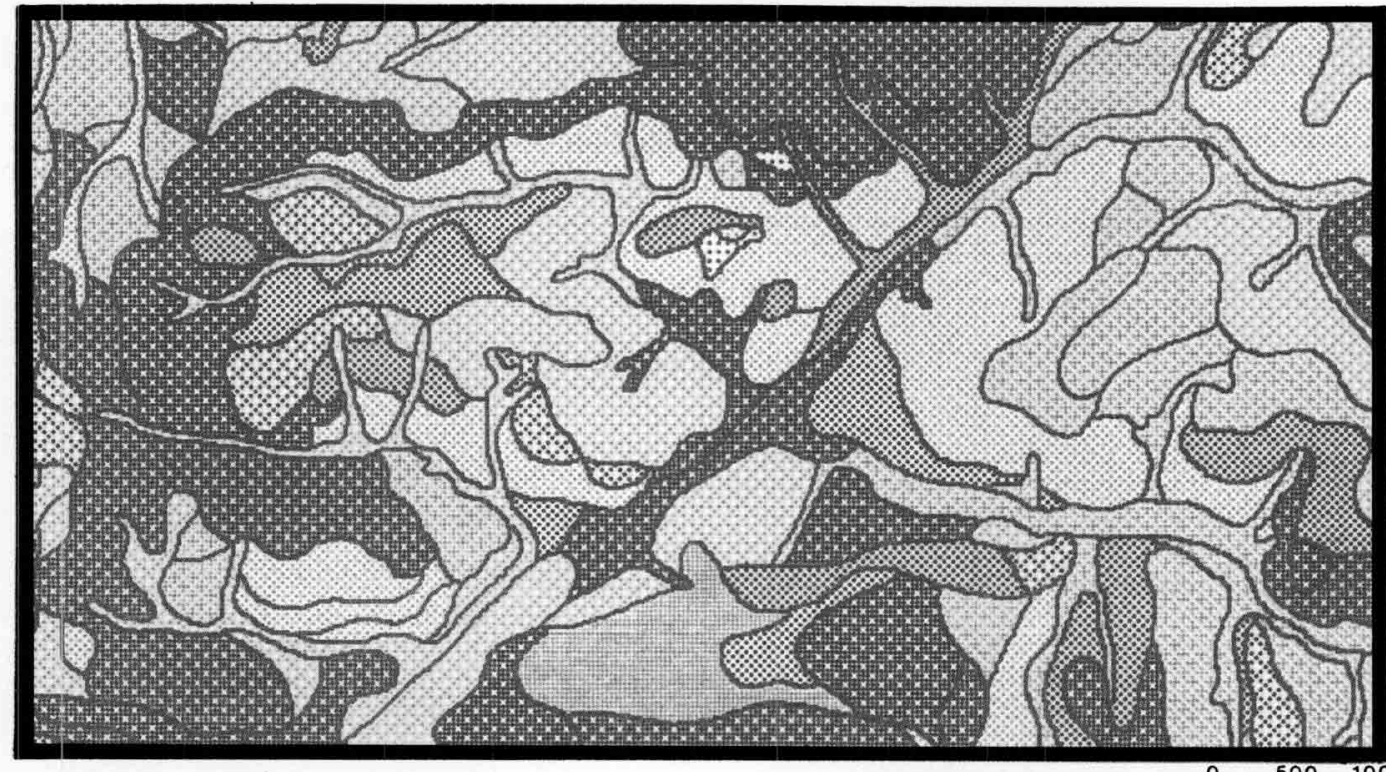

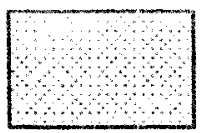

1

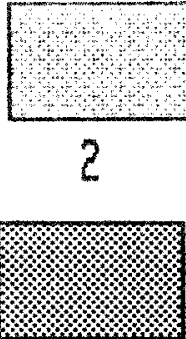

8

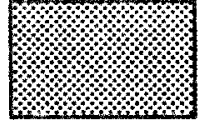

3

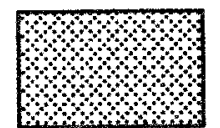

9

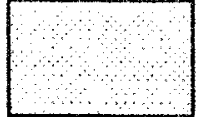

4

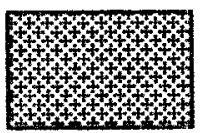

10

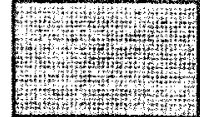

5

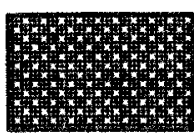

11

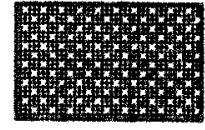

6

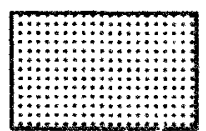

12

Figure 4

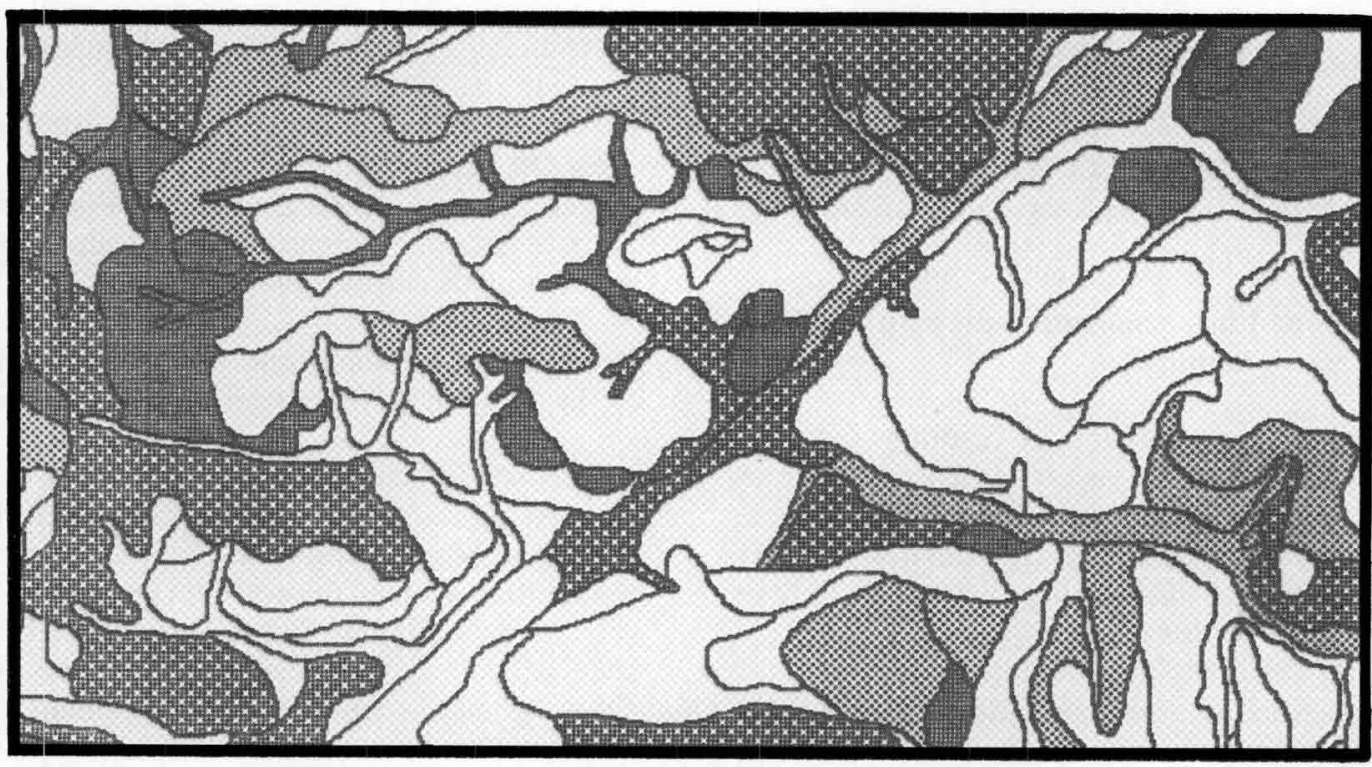

$5001000 \mathrm{~m}$

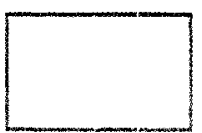

1

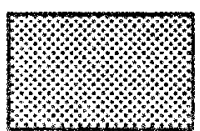

2

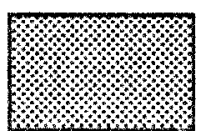

3

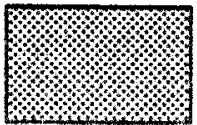

4

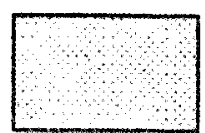

5

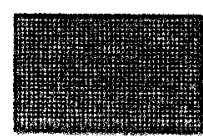

6

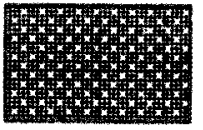

7 
L'exemple de la carte de la "réserve en eau des sols » est caractéristique de la mise en place de modèles résultant de la combinaison de caractères descriptifs du sol. Dans ce cas précis, ce modèle a été établi au vu des relations statistiques existant entre, d'une part, la réserve utile calculée à partir de la densité apparente, des teneurs pondérales en eau à la capacité de rétention et au point de flétrissement, et, d'autre part, de caractères immédiatement perceptibles en cartographie : texture, matière organique, charge en éléments grossiers... Mais notre propos n'est pas de développer les modèles possibles de calcul de la réserve en eau du sol à partir de ces caractères immédiats, mais de montrer l'intérêt d'un tel système informatique. Ainsi en visualisant l'extension spatiale des résultats, il nous est possible de perfectionner un modèle en le complétant chaque fois davantage de façon à ce qu'il coïncide au mieux avec les résultats localisés de mesures quantitatives précises.

\section{Propositions d'un classement des différents types de variations pédologiques}

L'utilisateur non spécialiste a trop souvent tendance à considérer l'unité cartographique de sols comme homogène. Sur de telles bases, la thématisation du document pédologique devient erronée.

En haut, figure 4

Carte de la texture de surface des sols du canton de Noyon (feuille $d u$ département de l'Oise à l'échelle du 1/50000). KALEIDOS-I.N.R.A.

Map of the surface texture of soils from the Noyon Canton (sheet of Oise department to scale 1/50 000). KALEIDOS-I.N.R.A.

\begin{tabular}{|c|c|c|c|}
\hline & & Sigles & \\
\hline Unité & $1:$ limon moyen & LI) & $531.08 \mathrm{ha}=18.7 \%$ \\
\hline Unité & 1 : limon moyen sableux & E1\} & 351,00 na $=10,7 \%$ \\
\hline Unité & 2 : limon argilo-sableux & E2) & $509,76 h a=17,9 \%$ \\
\hline Unité & 2: limon argileux & $L 2\}$ & \\
\hline Unité & $3:$ limon sableux & $F I)$ & $180,90 \mathrm{ha}=6,4$ \\
\hline Unité & $3:$ limon sablo-argileux & $F 2\}$ & $100,90 \mathrm{na}=0,4$ \\
\hline Unité & $4:$ argile limoneuse & $A I)$ & $458.31 \mathrm{~h} a=16,1 \%$ \\
\hline Unité & 4 : argile & $A 2\}$ & \\
\hline Unité & 5 : argile lourde & $O$ & $81,92 h a=2,9 \%$ \\
\hline Unité & 6: argile sableuse & $U$ & $26,65 h a=$ \\
\hline Unité & 7 : sable & $S$ & $281,03 \mathrm{ha}=9,9 \%$ \\
\hline Unité & $8:$ sable argileux & $Y$ & $119,29 h a=4,2 \%$ \\
\hline Unité & $9:$ sable limoneux & $I$ & $102,16 \mathrm{ha}=3,6 \%$ \\
\hline Unité & $10:$ tourbe & $T$ & $00 h a=$ \\
\hline Unité & 11 : unités complexes & $\begin{array}{l}\text { Total } \\
\text { complexes }\end{array}$ & $549,26 h a=19,3 \%$ \\
\hline$U$ & $: z$ & & $6,02 h a=$ \\
\hline
\end{tabular}

En bas, figure 5

Carte de la variabilité intra-unité de la texture de surface des sols du canton de Noyon feuille du département de l'Oise à l'échelle du I/50 000). KALEIDOS-I.N.R.A.

Map of intra-unit variability of soil surface texture from the Noyon canton (map of Oise department 10 scale 1/50000). KALEIDOS-I.N.R.A.

Unité 1 : pas de variabilité

Unité 2 : var. selon la pente

Unité 3 : var. progressive

Unité 4 : var. au hasard

Unité 5 : substrats géologiques

Unité 6 : hésitation entre classes

Unité 7 : complexes de sols

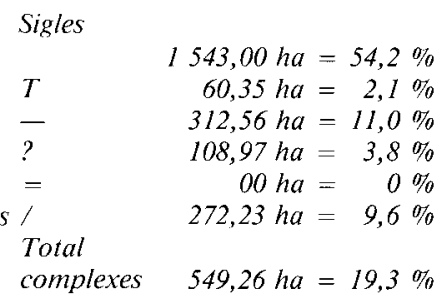

Les essais de traitements informatiques présentés précédemment nous ont permis de préciser cette notion de l'homogénéité intra-unité et de compléter la fiche de description des unités de sols par des possibilités d'expression de la variabilité du sol. Nous proposons ainsi une typologie pour les différentes variations rencontrées (il s'agit des variations pédologiques qui ne sont pas elles-mêmes exprimables sous la forme d'unités cartographiques individualisées par un contour).

\section{Variations de type aléatoire : limite de précision cartographique}

On décrit une unité de sols en grande partie par des caractères relevés à partir de quelques pédons (échantillonnage au sens statistique). On estime ainsi pour chacun de ces caractères, leur fonction de répartition.

Dans le cas le plus simple, cette fonction présente un maximum (ou mode) autour duquel se répartissent des individus dont le nombre diminue à mesure que l'on s'éloigne de ce point central (notion d'écart type, de périmode et de coefficient de dissymétrie, GIRARD, 1983).

L'écart autour d'un point central est plus ou moins important. S'il est très petit, aucun problème ne se pose. S'il est assez grand, il est nécessaire de savoir si la variabilité observée est du type aléatoire (par exemple variation inexpliquée du caractère sur de courtes distances géographiques) ou si elle est déterminée par des facteurs pédogénétiques éventuellement cartographiables à de très grandes échelles.

Le terme " aléatoire » ne signifie pas que la distribution des sols se fait au hasard mais que le phénomène nous apparaît d'une façon aléatoire au vu du nombre d'observations et des connaissances que nous possédons. Nous ne sommes pas en mesure de donner la (ou les) loi(s) de répartition des sols à l'intérieur de l'unité cartographique et donc encore moins de tracer des ébauches de contours. Nous nous situons aux limites de la précision cartographique que nous nous fixons en relation avec l'échelle de travail. Nous pourrions donner simplement la fonction de répartition de chacun des caractères de l'unité de sol mais cela demanderait également de nombreuses observations et donc une cartographie plus fine. Une approximation des valeurs des paramètres statistiques caractéristiques de cette fonction de répartition pourrait suffire (tel(s) le(s) mode(s), le périmode ou le coefficient de dissymétrie).

En réalité, cette dernière façon de procéder est également difficile à appliquer : d'une part, les caractères sont souvent qualitatifs (ou la fonction n'est pas unimodale continue) et, d'autre part, le nombre d'observation est insuffisant pour apprécier de tels paramètres statistiques. Nous choisissons alors des valeurs de classes dont les seuils sont fixés pour des raisons pédologiques ou agronomiques. Le mode est estimé par la valeur médiane d'une classe et la variation par l'étendue de cette classe.

Suivant la nature des terrains, l'auteur de la carte est en mesure de donner une étendue de classe plus ou moins large. Pour cette raison, nous proposons de faire un choix dans un niveau de précision correspondant à un nombre de classes plus ou moins important. Par exemple, pour le caractère profondeur, nous proposons une étendue de la classe qui soit de 5 , de 10 ou de $40 \mathrm{~cm}$. Si l'on est en présence d'une extrême varia- 


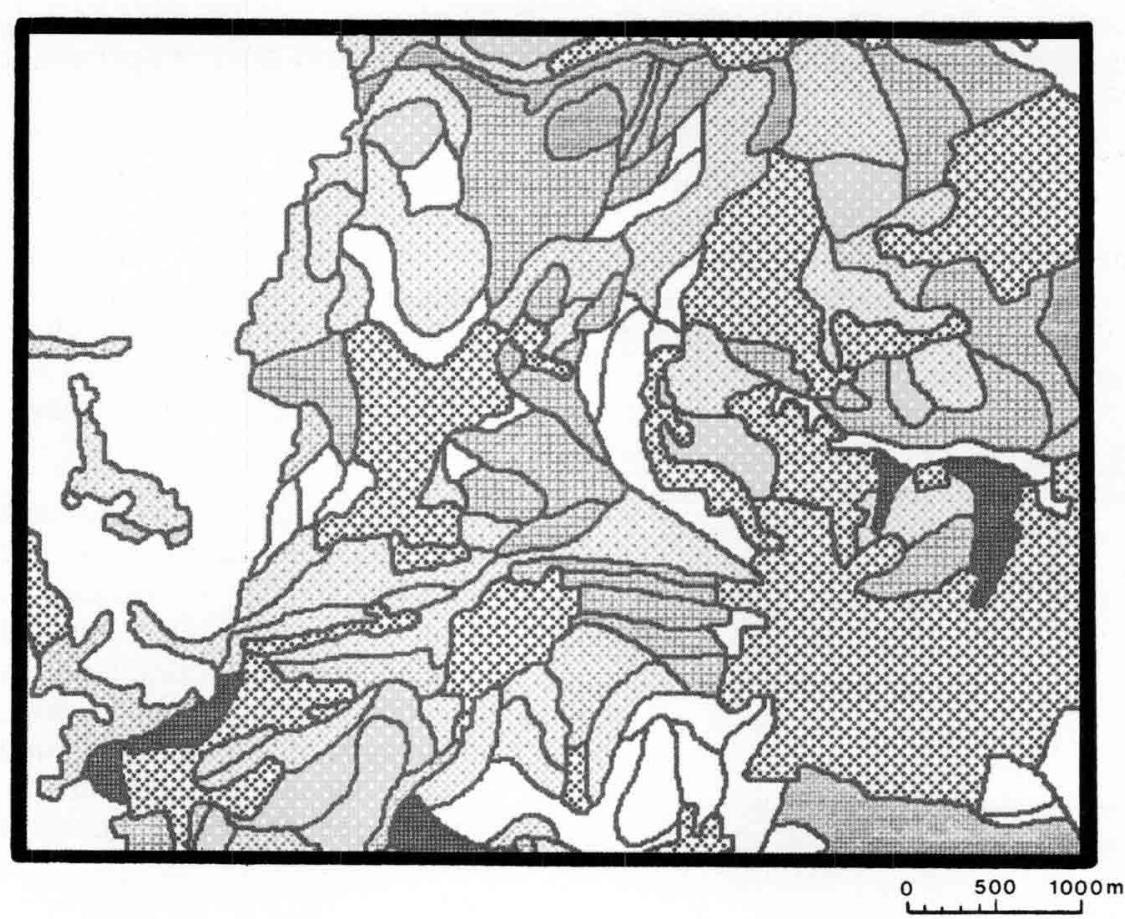

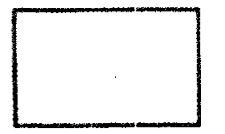

1

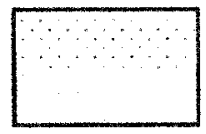

2

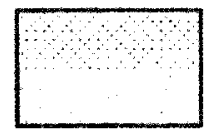

3

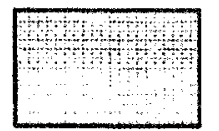

4

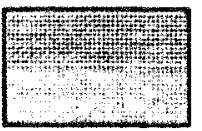

5

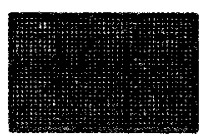

6

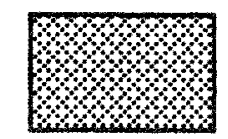

7

Figure 6

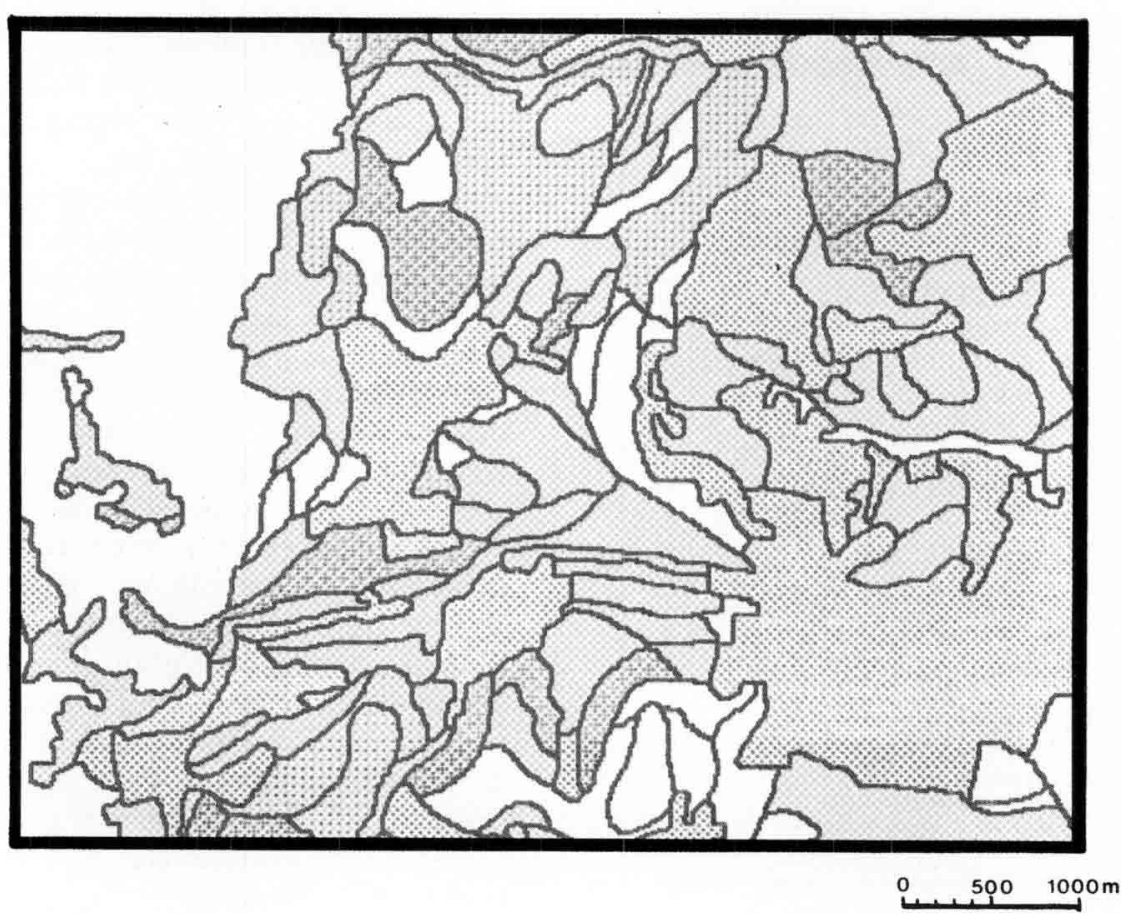

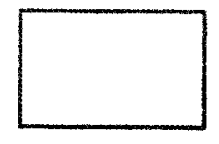

1
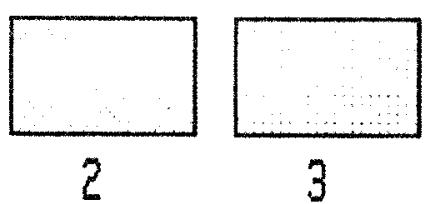

3

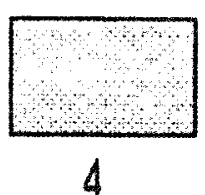

Figure 7 
En haut, figure 6

Carte de la réserve en eau des sols de la feuille de Molsheim à l'échelle du 1/50000.

Map of water tank soils of the Molsheim sheet to scale 1/50 000.

Unité $1:$ R.U. $<75$

Unité $2: 75<$ R.U. $<150$

Unité $3: 150<R . U .<200$

Unité $4: 200<R . U .<240$

Unité $5: R . U .>240$

Unité $6: R$.U. inconnue

Unité $7:$ zones non agricoles

En bas, figure 7

Carte de la variabilité intra-unité de la réserve en eau des sols de la feuille de Molsheim à l'échelle du 1/50 000.

Map of intra-unit variability of the water tank soils of Molsheim sheet to scale $1 / 50000$.

Unité 1 : pas de var. reconnue

Unité $2: R$.U. non calculée

Unité 3 : Var. entre $10 \& 50 \mathrm{~mm}$

Unité 4 : var. entre $50 \& 100 \mathrm{~mm}$

bilité (ou bien sûr d'une absence d'information), il vaut mieux n'inscrire aucune donnée. Dans l'objectif d'une thématisation future, la donnée manquante est préférable à une estimation hasardeuse.

\section{Variations de type déterministe}

Ce sont des variations dont on connaît les facteurs déterministes ou tout au moins les lois cartographiques de répartition. Dans ce cas, ce n'est plus seulement la valeur modale qui caractérise la fonction de répartition, mais également les extrêmes.

Nous avons regroupé les fonctions de répartition en quelques grands types suivant que la variation atteint un nombre limité de caractères ou concerne tous les caractères.

Dans le $1^{\text {er }}$ cas, nous avons prévu de noter sur une même fiche les extrêmes de cette variation ainsi que la nature de leur liaison.

Par exemple, "LATAL » = signifie «Texture limono-argileuse (LA) passant le long de la pente à une texture argilo-limoneuse (AL)»; le sigle « $\mathrm{T} »$ exprime un type de variation.

Les différents sigles utilisés peuvent traduire une répartition bimodale du caractère, ce dernier prenant, au sein de l'unité de sol, 2 valeurs contrastées avec peu d'intermédiaires. Au contraire, nous pouvons avoir une répartition unimodale avec un mode très large exprimant que le caractère prend différentes valeurs situées entre 2 extrêmes. Les exemples de la figure 8 sont théoriques mais nous voulons, par cette méthode, permettre de préciser si la variation intra-unité est de type net ou de type continu. Nous proposons une liste non exhaustive de sigles (fig. 9) qui peut être complétée, suivant les régions cartographiées, par différents types de variations caractéristiques de ces régions et expliquant au mieux la mise en place et le fonctionnement des sols (KING, 1982).

Dans d'autres cas, les variations concernent tous les caractères descriptifs : on est en présence de plusieurs unités de sol et on a recours à la notion de complexe de
Cas d'une unité de sol avec un paramètre prenant deux valeurs contrastées sans intermédiaires.
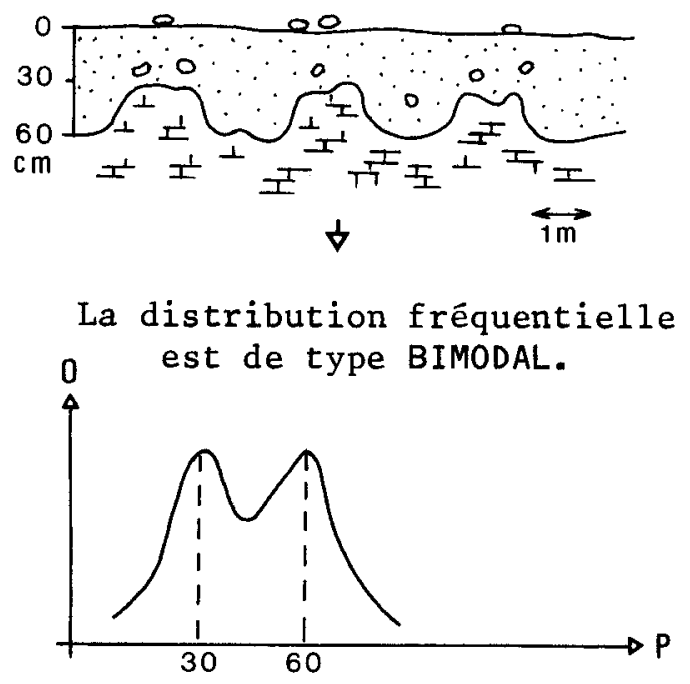

Cas d'une unité de sol avec un paramètre prenant uniformément des valeurs comprises entre deux extrêmes.

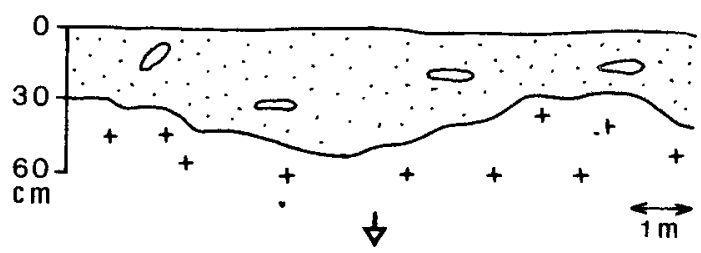

La distribution fréquentielle

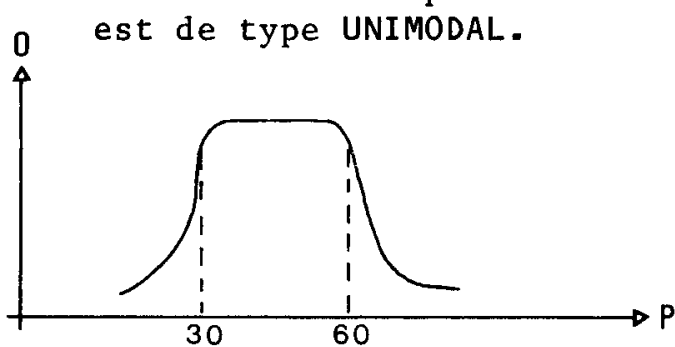

0 : \% d'observations ayant la profondeur $n_{\mathrm{cm}}$

$\mathrm{P}$ : profondeur en $\mathrm{cm}$.

Figure 8

Exemples théoriques de la variabilité intra-unité du caractère « profondeur du sol ".
Theoretical example of intra-unit variability of the « soil depth» characteristic. 
Variation de type bimodal

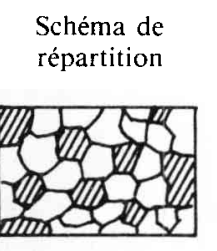

Disposition en mosaïque

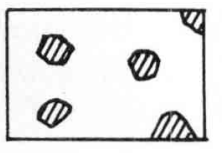

Disposition en cellules

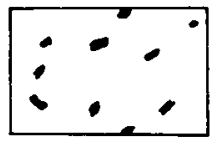

Disposition en taches régulières (< $10 \%$ du total) $+$

0

Sigle pour encodage

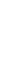

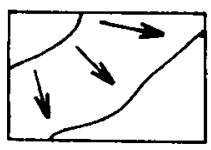

Variation de type unimodal

$$
\text { Type de répartition }
$$

Sigle pour encodage

Phénomènes d'ondulation du caractère. Celui-ci prend toutes les valeurs comprises entre 2 W extrêmes

Idem au précédent mais l'orientation est due à la pente

Passage progressif orienté géographiquement (ex.: changement de faciès du substrat)
$?$

$\mathrm{F}$

Figure 9

Quelques types de répartitions possibles de caractères du sol au sein d'une unité cartographique.

sols; il faut, dans ce cas, décrire autant de fiches qu'il y a d'unités de sols au sein du complexe.

De la même façon, les liaisons entre unités de sols au sein d'un même complexe ont été définies et codées (d'après FrIDLAND, 1976; ROZHKOV \& FRIDLAND, 1977 ; BOULA.INE, 1980).

Les unités de sols appartenant à un même complexe de sols et dont on ne connaît pas les lois de répartition ont été notées en association. A l'inverse, si on peut fournir des éléments sur le type d'organisation spatiale existant entre ces unités, on note la présence d'une combinaison. Dans ce dernier cas, plusieurs paramètres ont été choisis pour préciser cette organisation : le type de relation spatiale (mosaïque, bandes concentriques, bandes orientées...), l'ordre de grandeur de la variation spatiale (micro, méso ou macro-combinaison), le pourcentage de surface de chacune des unités de sols par rapport au complexe (variable nommée recouvrement), le degré d'éloignement taxonomique des unités (contraste), le type de transition (courbure).

\section{Validité des cartes produites}

Par une informatisation des cartes pédologiques, nous pensons diminuer la perte d'information et donc aboutir à des documents thématiques plus précis et plus complets. L'automatisation permet la production de nombreux documents d'une façon simple et dans des délais très brefs. Cela pose d'une façon plus cruciale le problème de la validité des documents fournis.

Some possible distributions of soil characteristics within a cartographic unit.

Il existe de nombreuses méthodes pour contrôler un document cartographique. On vérifie pour cela soit l'emplacement de limites pédologiques, soit la caractérisation des unités cartographiques (BIE \& BECKETT, $1971 a, b$; CAMPBELL, 1977 ; Legros, 1978 $a, b$; BURROUGH \& KOOL, 1981).

Il est important de connaître la nature de ce que l'on veut vérifier : appartenance des sols à une même classe d'une taxonomie de référence, valeur d'un ou de plusieurs caractères du sol... Ces méthodes font appel le plus souvent à une nouvelle phase de prospection, soit à une plus grande échelle sur des zones-tests représentatives, soit par un semis de points placés au hasard. Toutes ces méthodes sont coûteuses et rarement expérimentées. Il est donc important de mettre en garde l'utilisateur de documents thématiques extraits de la carte pédologique contre une interprétation trop rapide de ces documents (par exemple en considérant toutes les unités de sols comme intrinsèquement homogènes). Il existe au sein d'une même région des zones de forte complexité que le pédologue sait très bien reconnaître. Par le jeu de la codification de la variabilité intra-unité proposée précédemment et par l'emploi des complexes de sols, nous pouvons indiquer les zones d'hétérogénéité non cartographiables à l'échelle du document. La codification de la variabilité intra-unité d'un caractère est traitée informatiquement d'une façon identique à ce caractère. Le programme nous dresse la liste des codes utilisés et nous pouvons visualiser cartographiquement l'importance et la localisation des zones hétérogènes. L'édition de cartes de variabilité (fig. 5 et 7) 
donne ainsi à l'utilisateur une première estimation de la fiabilité qu'il pourra accorder aux documents thématiques.

\section{CONCLUSION}

Les techniques informatiques, par leur rapidité et leur fiabilité, sont capables d'automatiser une partie du travail cartographique. En cela, elles ouvrent de nouvelles possibilités encore inexplorées. C'est le cas de l'édition automatique de toutes sortes de documents dérivés de la carte pédologique. La méthode présentée n'enlève rien à l'édition classique de ce document puisqu'il est possible de mémoriser et d'exprimer aussi bien des données de base qu'une information synthétique plus générale. Elle permet de supprimer les délais d'attente de l'impression classique, de diminuer les coûts de production et de valoriser ainsi le document pédologique.

La carte des sols n'est qu'une expression partielle d'une réalité complexe. Toute carte thématique ne pourra donc être le reflet exact de cette réalité. De plus, il est important de souligner que les thèmes traités correspondent rarement d'une façon directe à un critère ayant servi au cours de l'élaboration cartographique. D'une part, les seuils employés par le pédologue, pour un critère quelconque, ne sont pas systématiquement identiques aux seuils désirés par le thématicien
(GIRARD, 1979), d'autre part, ce dernier s'intéresse souvent à des problèmes de fonctionnement ou de comportement des sols ; d'où la nécessité de mettre en place des modèles combinant les différents caractères de base. La méthode proposée permet de construire aisément toutes sortes de modèles plus ou moins complexes, mais cette facilité ne doit pas nous cacher le problème de la fiabilité que l'on peut accorder aux caractères descriptifs de base de la carte des sols et, par voie de conséquences, aux résultats thématiques. C'est pourquoi nous proposons l'édition systématique de cartes, dites de la variabilité intra-unité de ces thèmes, mettant en garde l'utilisateur sur la validité des documents produits.

Enfin, le thématicien fait appel à d'autres types de données cartographiques : climat, végétation, topographie, occupation du sol, géologie... etc. Nous nous sommes volontairement limité au seul document pédologique bien que la superposition par voie informatique de ces divers documents soit techniquement réalisable par KALEIDOS. Il reste, néanmoins, à construire des algorithmes capables d'éditer des documents de synthèse entre toutes les sources d'information, souvent cartographiées à des échelles et selon des méthodes très différentes.
A.C.C.T. (Agence de coopération culturelle et technique), 1979. Normalisation et échange de données pédologiques par ordinateur, ACCT, Paris, $192 \mathrm{p}$.

Aubry A. M., Boulvert Y., Séchet P., 1980. Un service offert aux pédologues : représentation automatique élaborée d'un caractère spatial. Cah. O.R.S.T.O.M., Sér. Pédol., 28 (3-4), 261-272.

Begon J. C., Hardy R., Mori A., Roque J., Jamagne M., 1977. Les sols du département de l'Oise. S.E.S.C.P.F.-I.N.R.A.-D.D.A. Oise, $333 \mathrm{p}$.

Bertrand R., Falipou P., Legros J. P., 1979. STIPA, notice pour l'entrée des descriptions et analyses de sols en banque de données. I.N.R.A.-I.R.A.T., 119 p.

Bie S. W., Beckett P. H. T., 1971 a. Quality control in soil survey. Introduction I. The choice of mapping unit. J. Soil Sci., 22 (1), 32-49.

Bie S. W., Beckett P. H. T., 1971b. Quality control in soil survey. II. The cost of soil survey. J. Soil Sci., 22 (4), 453-465.

Bie S. W., Schelling J., 1977. An integrated information system for soil survey. 43-49. In A. L. Sadovski \& S. W. Die. Proc. 2nd Meet. WGSIS, Varnia, Bulgaria. Pudoc, 113 p.

Boon W., 1981. Note brève concernant le développement d'un système informatique des données de sol en Belgique. 19-27. Sols, 4,

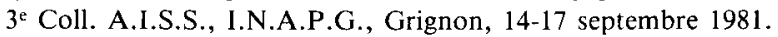

Boulaine J., 1980. Pédologie appliquée. MASSON Paris (coll. «Sciences agronomiques »), $220 \mathrm{p}$.

Burrough P. A., 1980. The development of a landscape information system in the Netherlands, based on a turn-key graphics system. Géo-processing, 1 (3), 257.

Burrough P. A., Kool J. B., 1981. A comparison of statistical techniques for estimating the spatial variability of soil properties in trial fields. 29-37. Sols, 4, $3^{\mathrm{e}}$ Coll. A.I.S.S., I.N.A.P.G., Grignon, 1417 septembre 1981 .
Campbell J. B., 1977. Variation of selected properties across a soil boundary. Soil Sci. Soc. Am. J., 41 (3), 578-582.

Cormack R. S., 1976. Data delineation and computer techniques for line printer mapping and tabulation. 58-66. In W. Moore \& S. W. Bie, eds. Proc. Meet. WGSIS, Canberra, Australia, Pudoc, $103 \mathrm{p}$.

C.P.C.S. (Commission de pédologie et de cartographie des sols), 1967. Classification des sols; commission de pédologie et de cartographie des sols. Doc. multi., I.N.R.A. Grignon, 96 p.

Cruickshand J. G., 1972. Soil Geography, David \& Charles, Newton Abbot, $225 \mathrm{p}$.

Domptail P., 1977. La cartographie automatique à la Société du canal de Provence et d'aménagement de la région provençale. Informatique et Biosphère. Actes $5^{e}$ coll., 113-143.

Dougenik A., Sheenan D. F., 1975. Symp user's reference manual. Laboratory for computer graphics and spatial analysis. Cambridge, Massachusetts.

Falque M., 1975. Méthode d'intégration des données du milieu naturel en matière d'aménagement de l'espace, 81-93. In "Informatique et biosphère ", étude et cartographie intégrée du milieu naturel, Paris, $142 \mathrm{p}$.

Ford G. L., Nielsen G. A., 1978. A computer graphic system for interactive display of land qualities and potentials. 11th Int. Congr. Soil Sci., 1, Abtr., p. 325. (Montana State University, Bozeman, Montana U.S.A.).

Fridland V. M., 1976. Levels of organisation of the soil mantle and regularities of soil geography. Cong. Int. Geogr., Moscou.

Garbouchev I. P., Sadovski A. N., 1978. Development and use of soil information systems in Europe. 11th Int. Congr. Soil Sci., 3, Symposium session papers, 132-142.

Girard M.-C., 1976. Recherche d'une méthodologie pédologique en matière de traitement statistique des données de sol. Applications à 
la taxonomie et à la cartographie. Bull. Assoc. Fr. Etude Sol, Sci. Sol, 3, 177-204.

Girard M.-C., 1979. Analyse des types de données pédologiques pour la cartographie. Informatique et Biosphère. Coll. Abidjan, 85106.

Girard M.-C., 1983. Recherche d'une modélisation en vue d'une représentation spatiale de la couverture pédologique. Thèse Doct. d'Etat. Sols, 12, 430 p.

Haans J. C. F. M., Van Lynden K. R., 1978. Assessment factors as an aid for interpreting soil surveys. Ilth Int. Congr. Soil Sci., 1, Abstr., p. 71

Hardy R., Roque J., Mettauer H., 1982. Carte départementale des terres agricoles. Molsheim., D.D.A.-I.N.R.A.-I.G.N.

Jamagne M., Begon J. C., Hardy R., 1977. La cartographie des sols, élément fondamental de l'aménagement et de la conservation de l'espace rural. Pédologie, 27 (1), 9-43.

Jansen I. J., Arnold R. W., 1976. Defining ranges of soil characteristics. Soil Sci. Soc. Am. J., 40, 89-92.

Johnson C. G., 1975. The role of automated cartography in soil survey, 48-51. In S. W. Bie, ed. Proc. Meet. WGSIS, Wageningen, the Netherlands. Pudoc, $87 \mathrm{p}$

King D., 1981. Deseription des unités de sols. Mémorisations et restitutions cartographiques. 41-57. Sols, 5, $3^{\mathrm{e}}$ Coll. A.I.S.S., I.N.A.P.G., Grignon, 14-17 septembre 1981.

King D., 1982. Description des unités cartographique de sols. Doc. poly., I.N.R.A.-S.E.S.C.P.F., 39 p.

King D., 1984. Exploitation informatique de cartes thématiques: Présentation du logiciel KALEIDOS de l'I.N.R.A. Sci. Géol. (à paraître).

Legros J. P., 1978a. Recherche et contrôle numérique de la précision en cartographie pédologique. 1. - Précision dans la délimitation des sols. Ann. Agron., 29 (5), 499-519.

Legros J. P., 1978b. Recherche et contrôle numérique de la précision en cartographie pédologique. 2. - Précision dans la caractérisation des unités de sols. Ann. Agron., 29 (6), 583-601.

Legros J. P., Hensel E., 1977. I.N.R.A. 6. Computer assisted cartographic system. 95-96. In A. L. Sadovski \& S. W. Bie, Proc. 2nd Meet. WGSIS, Vurnia, Bulgaria. Pudoc, 113 p.
Lynch L. G., 1976. Input methods and facilities available for land survey data, 11-18. In W. Moore \& W. Bie, Proc. Meet. WGSIS Canberra, Australia. Pudoc, $103 \mathrm{p}$

Mc Cormack D. E., Moore A. W., Dumanski J., 1978. A review of soil information systems in Canada, the United States and Australia. Itth Int. Congr. Soil Sci., 3, Symposium session papers, 143-160.

Mallet J. L., 1976. Programmes de cartographie automatique. Bibliothèque CARTOLAB. Sciences de la terre, 7, Inf. Géol.

Ragg J. M., 1977. Some forms of output from a soil survey information system. 1lth Int. Congr. Soil Sci., 1, Abstr., p. 40.

Rogala J.-P., 1982. Analyse de l'hétérogénéité et de la structure des unités cartographiques. Thèse Doct.-Ing. I.N.A. Paris, Grignon, $224 \mathrm{p}$.

Rozhkov V. A., Fridland V. M., 1977. Levels of the soil information systems organization according to the type of data, 30-36. In A. L. Sadovski \& S. W. Bie, Proc. 2nd Meet. WGSIS, Varnia, Bulgaria. Pudoc., $113 \mathrm{p}$

Tomlinson R. F., 1978. Design considerations for digital soil maps systems. IIth Int. Congr. Soil Sci., 3, Symposium session papers, 191-207.

Truszkowska R., Tokarski R., Wolszczak L., 1981. Data file of natural environment in a small scale description as a Polish soil information subsystem-Bigleb, 87-96. Sols, 4, $3^{\text {e }}$ Coll. A.I.S.S., I.N.A.P.G., Grignon, 14-17 septembre 1981.

Van den Driessche R. G., 1974. La banque de données pédologiques de I'O.R.S.T.O.M. Cah. O.R.S.T.O.M., sér. Pédol., 12 (1), 125132

Van Kuilenburg J., Bunschoten B., Burrough P. A., Schelling J., 1981. The digital soil map, scale $1: 50000$ of the Netherlands, 73-86. Sols, 5, $3^{e}$ Coll. A.I.S.S., I.N.A.P.G., Grignon, 14-17 septembre 1981.

Van Zuijlen L., 1975. Automation in cartography, 52-60. In S. W. BII, Proc. Meet. WGSIS, Wageningen, the Netherlands. Pudoc, $87 \mathrm{p}$.

Voss H. H., 1981. Soil data : capture, handling and display using the system DASP, 97-100. Sols, 4, $3^{\text {e }}$ Coll. A.I.S.S., I.N.A.P.G., Grignon, 14-17 septembre 1981 